\title{
Research article \\ Role of systemic inflammatory markers (biomarkers) as important prognostic factors for survival in head and neck cancer
}

\author{
Sumana Maiti Das ${ }^{1}$, Dharmendra Singh ${ }^{2}$, Anjana Bose (Ghosh Dastidar) ${ }^{3}$, Siddhartha Das ${ }^{4}$, Neena Prasad S. ${ }^{5}$, Aloke \\ Ghosh Dastidar ${ }^{6}$ Research, Kolkata, 700020, West Bengal, India
${ }^{2}$ Senior Resident, All India Institute of Medical Sciences, Patna, Bihar, India
${ }^{3}$ Associate Professor, Dept. of Anesthesiology, ${ }^{4}$ Professor, Dept. of Physiology, Diamond Harbour Govt. Medical College
Hospital, Diamond Harbour, South 24 Parganas, West Bengal, 743331, India \\ ${ }^{1}$ Assistant Professor, ${ }^{5}$ Junior Resident, ${ }^{6}$ Professor, Dept. of Radio-Therapy, Institute of Post-Graduate Medical Education and
}

\author{
(Received: November $2020 \quad$ Revised: October $2021 \quad$ Accepted: November 2021)
}

Corresponding author: Siddhartha Das. Email: das.siddhartha01@gmail.com

\begin{abstract}
Introduction and Aim: Cancer related inflammation is recognized as a critical multifaceted player in tumour initiation and progression. The neutrophil to lymphocyte ratio (NLR) and platelet to lymphocyte ratio (PLR) are important markers of systemic inflammatory burden in malignancy. The present retrospective study aimed to evaluate the prognostic value of pre-treatment baseline (NLR) and (PLR) for survival in HNC patients.

Materials and Methods: Analysis of data of 257 patients with head and neck cancer treated with definitive therapy over 3.5 yrs. Neutrophil, lymphocyte and platelet counts before treatment of all patients were collected and NLR and PLR were calculated.

Results: Median value of NLR and PLR were 3.7 and 170.63 respectively. Receiver operator characteristics (ROC) curve analysis showed the predictive cut-off value of NLR and PLR as 3.9 and 158.3. Univariate analysis using Cox-regression model showed NLR $(p<0.001)$ and PLR $(p=0.001)$ significantly influenced the locoregional recurrence free survival (LRRFS) and overall survival (OS). The multivariate analysis showed NLR is the independent prognostic factors influencing LRRFS $(p=0.007)$ and OS $(p=0.002)$. Kaplan-Meier survival curve (Log rank test; Chi-square $\left(\chi^{2}\right)$ value) showed OS is significantly influenced by NLR group (LRRFS; $\chi^{2}=23.9$ and OS; $\chi^{2}=33.7$ ) and PLR group (LRRFS; $\chi^{2}=11.2$ and OS; $\left.\chi^{2}=19.3\right)$ in contrast to LRRFS.
\end{abstract}

Conclusion: NLR can be strongly used as biomarker for prognostication for outcome and survival in head neck cancer. However, a well-designed, larger studies with longer follow-up is warranted.

Keywords: NLR; PLR; prognostic factors; head neck cancer (HNC).

\section{INTRODUCTION}

$\mathrm{H}$ ead and neck cancers (HNCs) affect the upper aerodigestive tract and are one of the most common cancers worldwide (1). Nearly $60 \%$ of the population presents with locally advanced but non-metastatic disease. Radiotherapy along with concurrent chemotherapy has long been the standard nonsurgical therapy for locally advanced disease. Definitive chemo-radiotherapy is the main modality of treatment in locally advanced cancer but response varied depending upon both tumour characteristics and host related factors like age, sex, performance status etc., (2).

Inflammation is considered to have role in tumour development and progression (3). In the $19^{\text {th }}$ century, Rudolf Virchow showed leukocytes were present in tumours and suggested a possible link between inflammation and cancer and chronic inflammation together with the tissue injury enhance cell proliferation (4). Various studies have also showed close associations of systemic inflammation as an independent prognostic factor for treatment outcomes for many types of cancers, including colorectal cancer, renal cell cancer, pancreatic cancer, and head and neck squamous cell carcinoma (5). Neutrophil to lymphocyte ratio (NLR) may also predict the survival in HNCs (6). Host response to malignant tumours involved in systemic alterations, not only changes in the tumour microenvironment. An elevation of circulating neutrophil count is thought to be the result of tumour cells releasing cytokines, which stimulate the bone marrow to produce neutrophils (7).

Malignant tumours may also influence the number of platelets and alter their functioning. Several hypotheses have been suggested on pathophysiological relation between tumour progression and thrombocytosis (8). Platelets may also support tumour progression through facilitation of neo-angiogenesis, generation of adhesion molecules and rise of early metastatic niches. For this reason, platelet to lymphocyte ratio (PLR) has been proposed as an independent prognostic factor in different solid tumours (9). Although various clinical and molecular biomarkers of treatment outcomes after definitive radiotherapy (RT) for HNC have been investigated, no clear consensus regarding reliable 
predictive indicators has been reached. This study aimed to prove NLR and PLR as a predictive biomarker for survival in HNCs.

\section{MATERIALS AND METHODS}

The clinical data of 257 patients treated either by definitive radiotherapy or by adjuvant radiotherapy at Institute of Post Graduate Medical Education and Research (IPGME\&R), Kolkata, from February 2015 to August 2018 were collected. Staging was done based on the American Joint Committee on Cancer (AJCC) TNM staging system (7th edition, 2010) (10). The diagnosis had been validated by histopathological examination. The inclusion criteria for the patients were as follows: (i) age more than 18 years; (ii) complete clinical, laboratory, imaging, and follow-up data, (iii) no chronic infectious diseases, (iv) without any haematological disorders or any treatment within one month before radiotherapy leading to elevated NLR and PLR, like administration of hematopoietic drugs such as GCSF, (v) no autoimmune disease or usage of steroids. The performance status of patients was categorised using Eastern cooperative oncology group (ECOG) (11). Routine blood counts were done within seven days prior to starting RT. NLR and PLR were calculated as the absolute neutrophil or platelet counts, respectively, divided by the absolute lymphocyte counts. After treatment completion, patients were followed up every 3 months. Local recurrence of the disease was referred as locoregional recurrence (LRR). The locoregional recurrence free survival (LRRFS) was defined as interval between completion of treatment (adjuvant radiation or definitive radiation) and locoregional recurrence. The overall survival (OS) was calculated as the interval between the completion of treatment to the date of death or the last visit.

\section{Statistical analysis}

The Statistical Package for the Social Sciences (IBM SPSS for Windows, version 25.0) was used for the statistical analysis. Descriptive statistics were used to characterize the patient population. Continuous variables were analysed using Student's t-test. Receiver operator characteristics (ROC) curve analysed using the NLR and PLR for locoregional recurrence and event at last follow-up to find out the predictive cut-off value of NLR and PLR. The assessment of the accuracy of NLR and PLR cut-off value is done by calculation of area under curve (AUC) and 95\% confidence interval (CI). The Kaplan-Meier method was used to compute LRRFS and OS. Comparison of variables influencing LRRFS and OS were done using Logrank test. Univariate and multivariate analysis were performed using Cox regression model. The factors found statistically significant on univariate analysis were included in multivariate analysis for survival outcomes. A pvalue less than 0.05 considered statistically significant in all performed analysis.

\section{RESULTS}

\section{Clinicopathological characteristics}

Two hundred and fifty-seven patients were included in this study. The epidemiological, clinical, pathological and therapeutic data have been mentioned in Table- 1 .

Table 1: Clinicopathological characteristics features of patients with head and neck carcinoma

\begin{tabular}{|c|c|c|c|}
\hline & & Count & Table N \% \\
\hline \multirow[t]{2}{*}{ Sex } & Male & 214 & $83.3 \%$ \\
\hline & Female & 43 & $16.7 \%$ \\
\hline \multirow[t]{6}{*}{ Age group } & $<30$ years & 2 & $0.8 \%$ \\
\hline & $31-40$ years & 21 & $8.2 \%$ \\
\hline & $41-50$ years & 49 & $19.1 \%$ \\
\hline & $51-60$ years & 87 & $33.9 \%$ \\
\hline & $61-70$ years & 73 & $28.4 \%$ \\
\hline & $>70$ years & 25 & $9.7 \%$ \\
\hline \multirow[t]{7}{*}{ Primary site } & CUP & 6 & $2.3 \%$ \\
\hline & Hypopharynx & 21 & $8.2 \%$ \\
\hline & Larynx & 97 & $37.7 \%$ \\
\hline & Nasopharynx & 6 & $2.3 \%$ \\
\hline & Oral cavity & 71 & $27.6 \%$ \\
\hline & Oropharynx & 50 & $19.5 \%$ \\
\hline & Sinonasal cavity & 6 & $2.3 \%$ \\
\hline \multirow[t]{8}{*}{ Symptoms } & Cough & 10 & $3.9 \%$ \\
\hline & Dysphagia & 44 & $17.1 \%$ \\
\hline & Epistaxis & 6 & $2.3 \%$ \\
\hline & Hoarseness of voice & 54 & $21.0 \%$ \\
\hline & Sensation of lump in throat & 9 & $3.5 \%$ \\
\hline & Non healing ulcer & 34 & $13.2 \%$ \\
\hline & Pain oral cavity & 51 & $19.8 \%$ \\
\hline & Swelling & 49 & $19.1 \%$ \\
\hline
\end{tabular}




\begin{tabular}{|c|c|c|c|}
\hline \multirow[t]{4}{*}{ ECOG PS } & ECOG 0 & 39 & $15.2 \%$ \\
\hline & ECOG 1 & 80 & $31.1 \%$ \\
\hline & ECOG 2 & 109 & $42.4 \%$ \\
\hline & ECOG 3 & 29 & $11.3 \%$ \\
\hline \multirow[t]{2}{*}{ Smoking } & No & 54 & $21.0 \%$ \\
\hline & Yes & 203 & $79.0 \%$ \\
\hline \multirow[t]{6}{*}{ Co-morbidity } & COPD & 6 & $2.3 \%$ \\
\hline & DM & 14 & $5.4 \%$ \\
\hline & HTN & 32 & $12.5 \%$ \\
\hline & Hypothyroidism & 3 & $1.2 \%$ \\
\hline & Seizure disorder & 2 & $0.8 \%$ \\
\hline & None & 200 & $77.8 \%$ \\
\hline \multirow[t]{2}{*}{ Surgery } & Yes & 45 & $17.5 \%$ \\
\hline & No & 212 & $82.5 \%$ \\
\hline \multirow[t]{5}{*}{ Stage group } & Stage I & 5 & $1.9 \%$ \\
\hline & Stage II & 10 & $3.9 \%$ \\
\hline & Stage III & 85 & $33.1 \%$ \\
\hline & Stage IVA & 114 & $44.4 \%$ \\
\hline & Stage IVB & 43 & $16.7 \%$ \\
\hline \multirow[t]{3}{*}{ Histopathology grade } & Well differentiated & 60 & $23.3 \%$ \\
\hline & Moderately differentiated & 166 & $64.6 \%$ \\
\hline & Poorly differentiated & 31 & $12.1 \%$ \\
\hline \multirow[t]{2}{*}{ Post-operative Surgical Margin } & Positive & 24 & $53.3 \%$ \\
\hline & Negative & 21 & $46.6 \%$ \\
\hline \multirow[t]{2}{*}{ NLR group } & Low risk NLR group. & 136 & $52.9 \%$ \\
\hline & High risk NLR group. & 121 & $47.1 \%$ \\
\hline \multirow[t]{2}{*}{ PLR group } & Low risk PLR group & 111 & $43.2 \%$ \\
\hline & High risk PLR group & 146 & $56.8 \%$ \\
\hline \multirow[t]{2}{*}{ Locoregional recurrence (LRR) } & Yes & 116 & $45.1 \%$ \\
\hline & No & 141 & $54.9 \%$ \\
\hline \multirow[t]{2}{*}{ Distant metastasis } & Yes & 16 & $6.2 \%$ \\
\hline & No & 241 & $93.8 \%$ \\
\hline
\end{tabular}

Different parameters including bio-markers with prognostic values have been mentioned in table 2 .

Table 2: Different parameters with prognostic values

\begin{tabular}{|c|c|c|c|c|}
\hline & Median & Maximum & Minimum & Standard Deviation \\
\hline Age (years) & 58 & 85 & 27 & 11 \\
\hline Duration of symptoms (months) & 5 & 36 & 1 & 6 \\
\hline Addiction duration (years) & 20 & 50 & 0 & 13 \\
\hline Hemoglobin (Hb) (g/dl) & 12.10 & 17.00 & 6.40 & 1.79 \\
\hline NLR & 3.70 & 9.44 & .49 & 1.76 \\
\hline Platelet count (lakhs/dl) & 3.00 & 9.10 & .90 & 1.32 \\
\hline PLR & 170.63 & 827.17 & 26.14 & 146.56 \\
\hline EBRT dose (Gy) & 66.0 & 66.0 & 4.0 & 11.61 \\
\hline Follow up duration (months) & 14 & 39 & 2 & 8 \\
\hline
\end{tabular}

\section{ROC curve and optimal cut-off value of NLR and PLR}

The optimal cut-off points of NLR was calculated for events (including locoregional recurrence and status at last follow-up) using ROC curve. The results showed that the optimal predictive cut-off value of NLR is 3.9 (area under ROC curve $=68.2 \%, \mathrm{p}<$ 0.001 ), at which most significant difference was observed in events. The sensitivity and specificity was $66.4 \%$ and $69.5 \%$ respectively (fig 1). The patients were divided into two groups based on NLR cut-off points into low risk NLR group (NLR $\leq 3.9)$ and high risk NLR group (NLR > 3.9). The frequency of distribution of NLR risk group i.e., low risk NLR and high risk NLR were $52.9 \%$ and $47.1 \%$ respectively. 


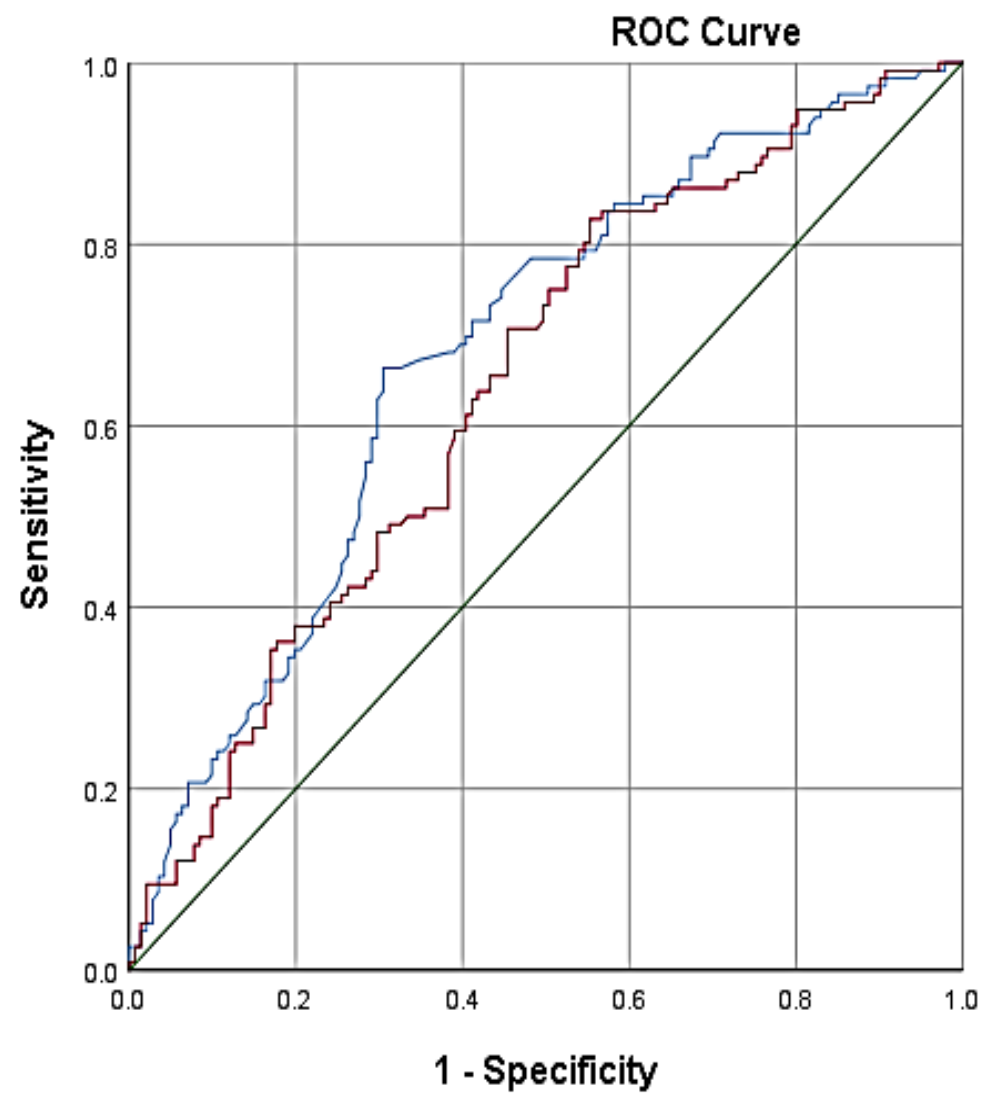

\section{Source of the} Curve

- NL RATIO

- PL RATIO

- Reference Line

Diagonal segments are produced by ties.

Fig. 1: The receiver operator characteristics curve of the cut-off point for NLR (blue curve); area under ROC curve $=68.2 \%$.

The receiver operator characteristics curve of the cut-off point for PLR (red curve); area under ROC curve $=64.4 \%$.

The optimal predictive cut-off value of PLR was 158.3 (area under ROC curve $=64.4 \%, \mathrm{p}<0.001$ ), at which most significant difference was observed in events. The sensitivity and specificity were $69.8 \%$ and $54.6 \%$ respectively (fig 1 ). The patients were divided into two groups based on PLR cut-off points into low-risk PLR group (PLR $\leq 158.3$ ) and high-risk PLR group (PLR > 158.3). The frequency of distribution of PLR risk group i.e., low risk PLR and high-risk PLR were $43.2 \%$ and $56.8 \%$ respectively.

\section{Prognostic factors affecting locoregional recurrence and overall survival}

The univariate analysis using the Cox-regression model showed thatECOG PS $(\mathrm{p}<0.001)$, smoking ( $\mathrm{p}$ $=0.006)$, stage of the disease $(p=0.007)$, grade of the tumour $(\mathrm{p}=0.041)$, NLR group $(\mathrm{p}<0.001)$ and PLR group $(\mathrm{p}=0.001)$ were statistically significantly influencing the LRR. The ECOG PS $(p<0.001)$, smoking status $(\mathrm{p}=0.020)$, stage of the disease $(\mathrm{p}=$ $0.019)$, NLR ( $\mathrm{p}<0.001)$, PLR $(\mathrm{p}<0.001)$ were statistically significantly influencing the OS (table 3a).

Table 3a: Univariate and multivariate analysis of factors associated with locoregional recurrence (LRR) in head and neck cancer

\begin{tabular}{|c|c|c|c|c|c|c|}
\hline Characteristics & \multicolumn{3}{|c|}{ Univariate analysis (LRR) } & \multicolumn{3}{c|}{ Multivariate analysis (LRR) } \\
\hline & HR & $95 \%$ CI & p-value & HR & $95 \%$ CI & p-value \\
\hline Age $<45$ & 1.058 & $0.652-1.715$ & 0.819 & & & \\
\hline ECOG & 1.903 & $1.526-2.373$ & $<0.001$ & 1.640 & $1.310-2.054$ & $<0.001$ \\
\hline Smoking & 2.094 & $1.231-3.561$ & 0.006 & 1.873 & $1.091-3.216$ & 0.023 \\
\hline Stage & 1.338 & $1.083-1.652$ & 0.007 & 1.217 & $0.970-1.528$ & 0.090 \\
\hline Grade & 0.720 & $0.525-0.987$ & 0.041 & 0.747 & $0.542-1.030$ & 0.075 \\
\hline Margin & 0.951 & $0.510-1.771$ & 0.874 & & & \\
\hline NLR group & 2.496 & $1.698-3.670$ & $<0.001$ & 2.010 & $1.205-3.350$ & 0.007 \\
\hline PLR group & 1.936 & $1.297-2.889$ & 0.001 & 1.030 & $0.604-1.759$ & 0.931 \\
\hline
\end{tabular}

The multivariate analysis showed that ECOG PS ( $\mathrm{p}<$ $0.001)$, smoking $(\mathrm{p}=0.023)$, and NLR group $(\mathrm{p}=$ $0.007)$ are the independent prognostic factors influencing LRR. The ECOG PS ( $\mathrm{p}<0.001)$, and
NLR ( $\mathrm{p}=0.002)$ are the independent prognostic factors influencing OS (table 3b). 
Das et al: Role of systemic inflammatory markers (biomarkers)

Table 3b: Univariate and multivariate analysis of factors associated with overall survival (OS) in head and neck cancer

\begin{tabular}{|c|c|c|c|c|c|c|}
\hline Characteristics & \multicolumn{3}{|c|}{ Univariate analysis (OS) } & \multicolumn{3}{c|}{ Multivariate analysis (OS) } \\
\hline & HR & $95 \%$ CI & p-value & HR & $95 \%$ CI & p-value \\
\hline Age $\leq 45$ years & 0.916 & $0.554-1.515$ & 0.733 & & & \\
\hline ECOG & 1.993 & $1.557-2.550$ & $<0.001$ & 1.716 & $1.336-2.205$ & $<0.001$ \\
\hline Smoking & 1.969 & $1.114-3.479$ & 0.020 & 1.753 & $0.964-3.023$ & 0.056 \\
\hline Stage & 1.316 & $1.046-1.656$ & 0.019 & 1.139 & $0.890-1.458$ & 0.301 \\
\hline Grade & 0.735 & $0.522-1.034$ & 0.077 & & & \\
\hline Margin & 0.773 & $0.413-1.449$ & 0.423 & & & \\
\hline NLR group & 3.443 & $2.192-5.376$ & $<0.001$ & 2.443 & $1.372-4.347$ & 0.002 \\
\hline PLR group & 2.474 & $1.708-4.419$ & $<0.001$ & 1.309 & $0.710-2.416$ & 0.389 \\
\hline
\end{tabular}

\section{Survival analysis}

The average follow-up duration of the study was 14 (2-39) months. A total of 116 patients had LRR and 16 patients developed distant metastasis and 98 patients died. The median LRRFS was 21 months
(95\% CI; 19.691 - 25.309; fig. 2). The median OS was 26 months (95\% CI; 19.453 - 32.547; fig. 3). 1 year and 3-year LRRFS was $67.6 \%$ and $28.5 \%$ respectively and 1-year and 3-year OS was $77 \%$ and $33 \%$ respectively.

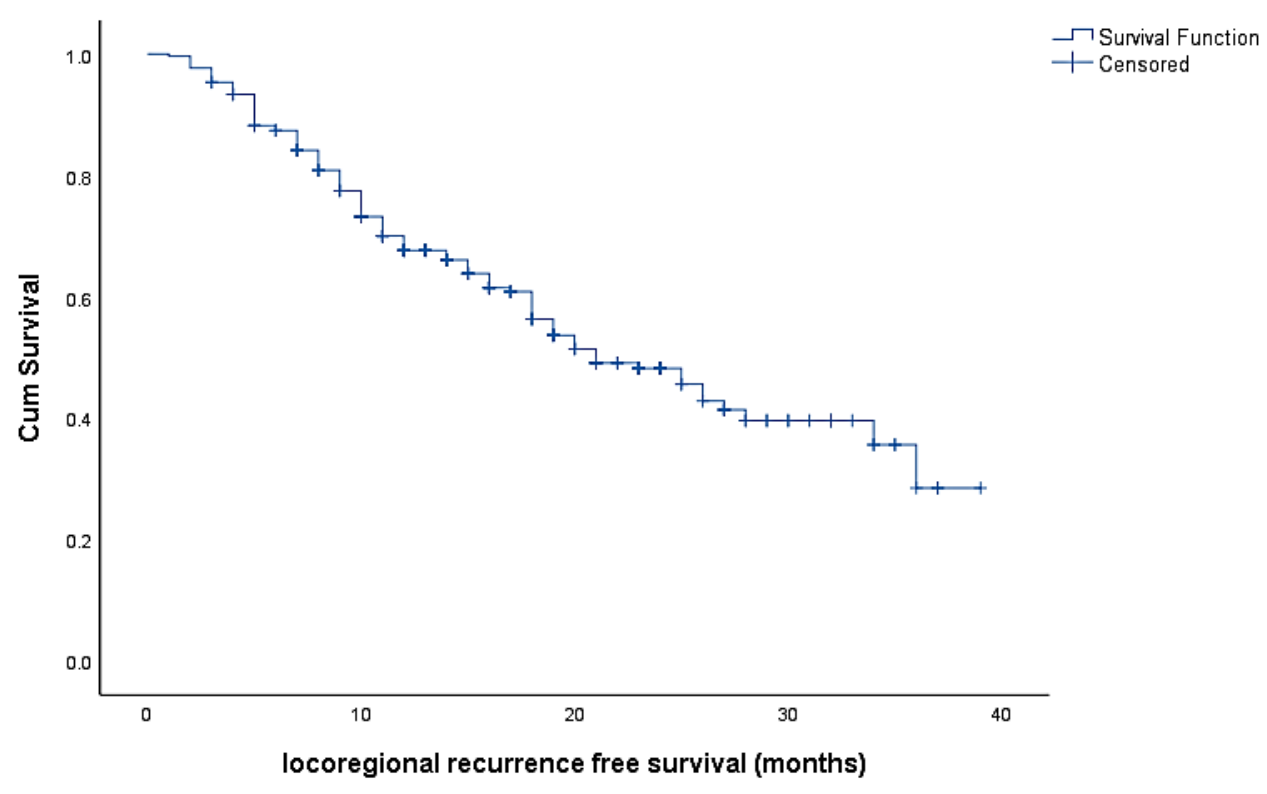

Fig. 2: The Kaplan Meier survival curve of locoregional recurrence free survival (LRRFS)

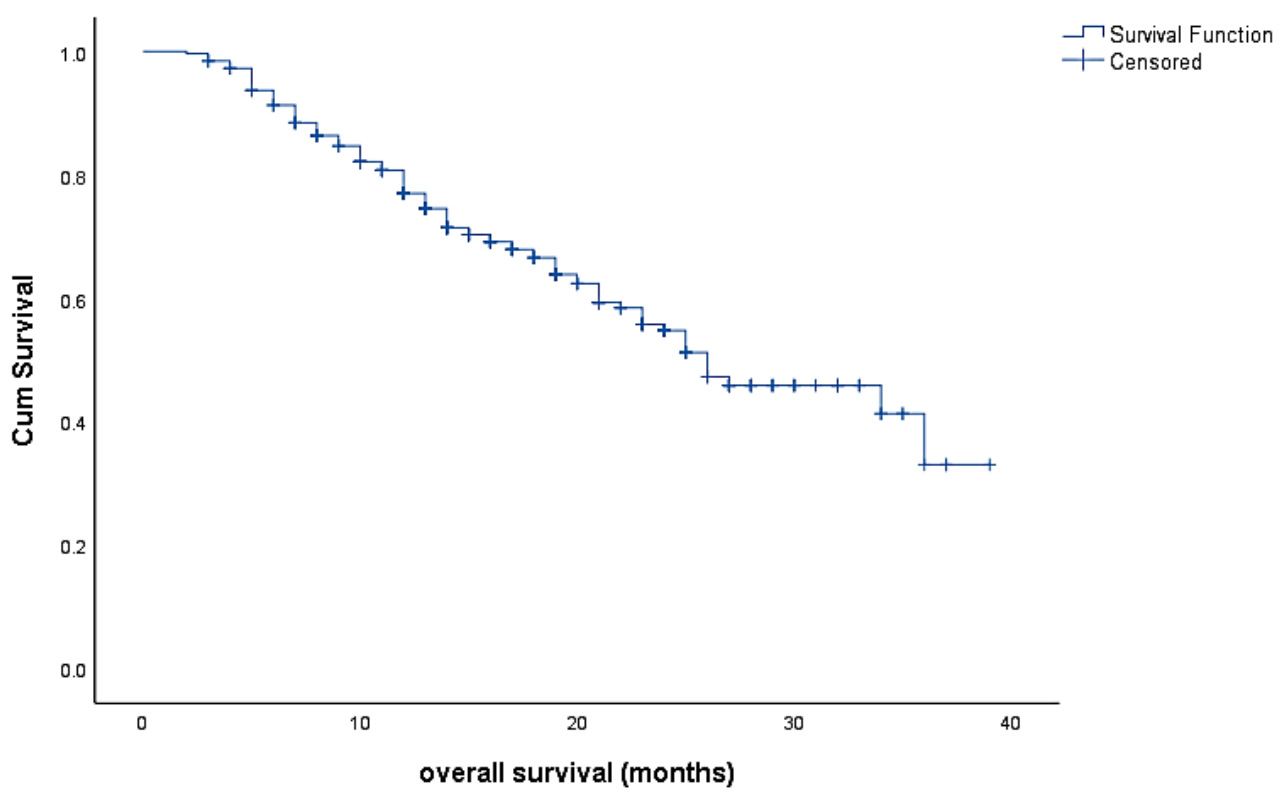

Fig. 3: The Kaplan Meier survival curve of overall survival (OS) 


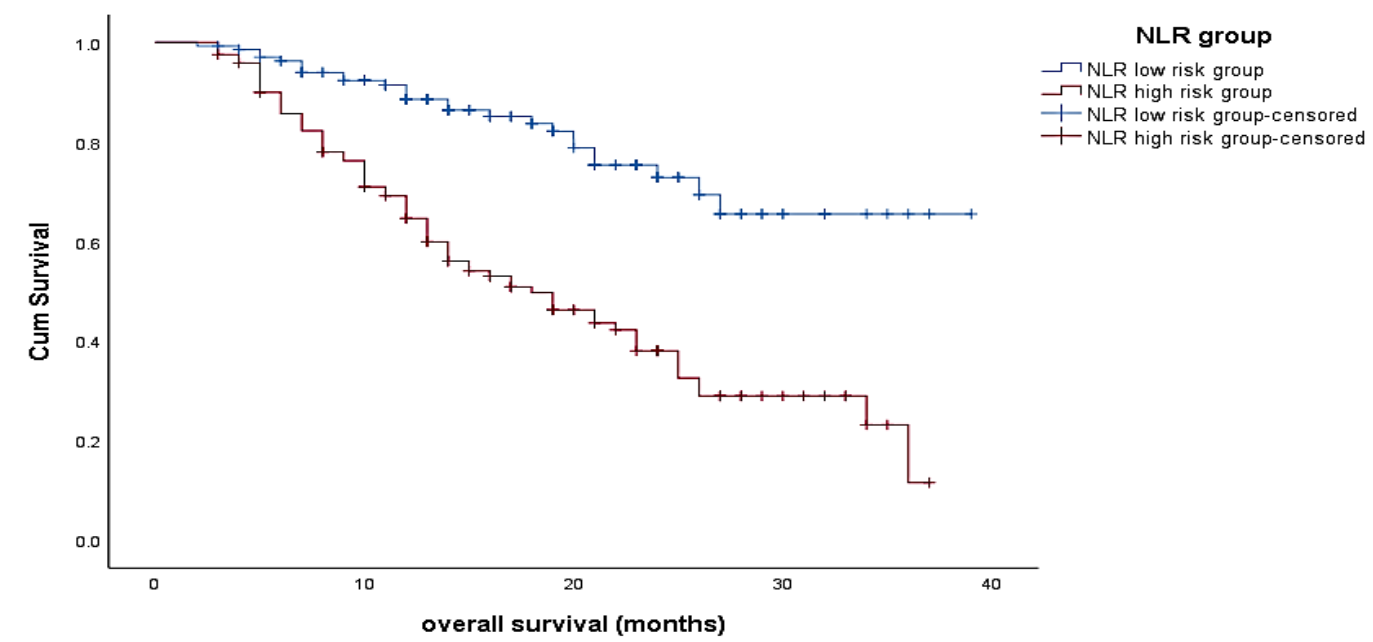

Fig. 4: The Kaplan Meier survival curve of OS according to NLR group; Log rank; $p<0.001$ and Chi-square value is 33.7

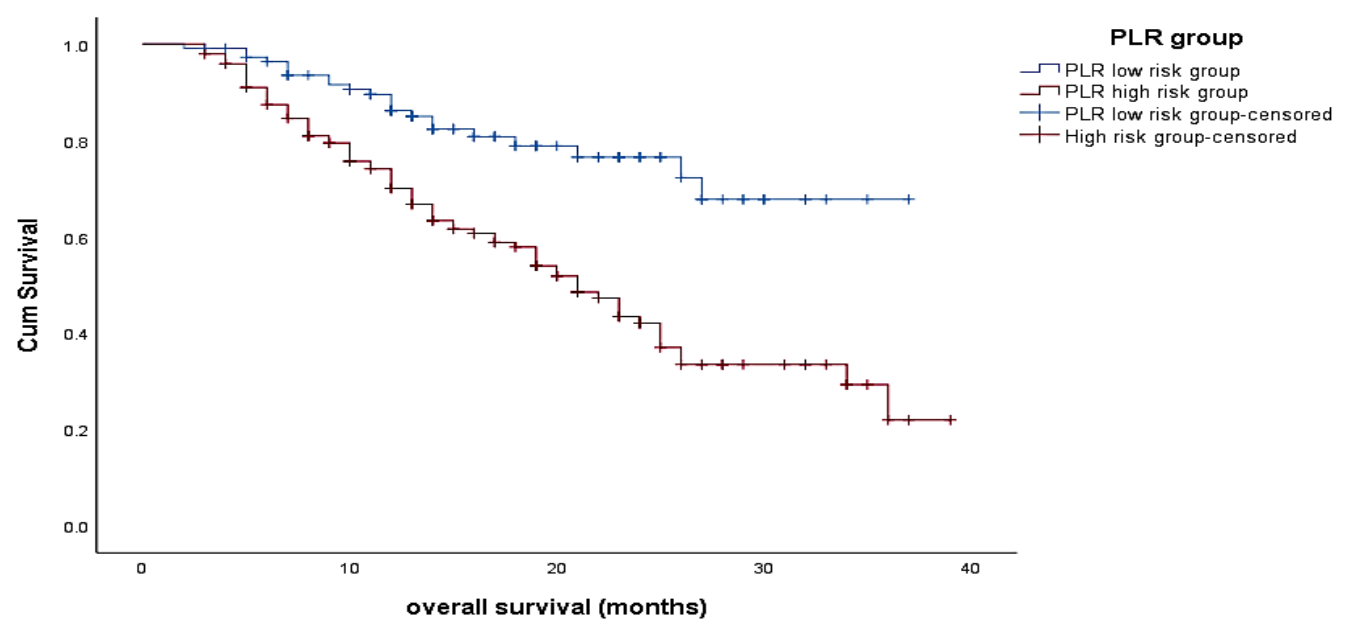

Fig. 5: The Kaplan Meier survival curve of OS according to PLR group; Log rank; $p<0.001$ and Chi-square value is 19.3

The LRRFS (Logrank test; $\mathrm{p}<0.001$ ) and OS (Logrank test; $p<0.001$ ) curves of NLR risk groups differed significantly. The median LRRFS and OS of low risk NLR group were not reached, while for high-risk group the median LRRFS and OS was 20.6 and 22.4 months respectively. The LRRFS (Logrank test; $\mathrm{p}<0.001)$ and OS (Logrank test; $\mathrm{p}<0.001$ ) curves of PLR risk groups were differing significantly. The median LRRFS and OS of low-risk PLR group were not reached, while for high-risk group the median LRRFS and OS was 18 months and 21 months respectively.

The Chi-square test value of NLR group for LRRFS $\left(\right.$ Log rank $\left.\chi^{2}=23.9 ; p<0.001\right)$ and OS (Log rank $\chi^{2}$ $=33.7$; $<<0.001$; fig. 4). LRRFS and OS influenced by NLR risk group as per Logrank test, but the Chisquare value of NLR risk group is more for OS compared to LRRFS. Therefore, it can be concluded that NLR risk group is influencing OS more than LRRFS. The Chi-square test value of PLR group for LRRFS (Log rank $\left.\chi^{2}=11.2 ; \mathrm{p}<0.001\right)$ and OS ( $\log$ rank $\chi^{2}=19.3 ; \mathrm{p}<0.001$; fig. 5). LRRFS and OS influenced by PLR risk group as per Logrank test, but the Chi-square value of PLR risk group is more for OS compared to LRRFS. Therefore, it can be concluded that PLR risk group is influencing OS more than LRRFS.

\section{DISCUSSION}

Inflammatory responses play major decisive roles at different stages of tumour development, including initiation, promotion, malignant conversion, invasion, and metastasis. Inflammation also affects immune surveillance and responses to therapy and elevated inflammatory mediators are related to poor prognosis in patients with cancer (12).

HNC occurs more frequently in older patients. The incidence of these tumours is low among the persons younger than 40 years. $9 \%$ of our patients were less than 40years. This rate matches with results of other studies around the world.Most of the patients (33.9\%) in our study were aged between 51 to 60 years and $80 \%$ patients were between 41 to 70 years of age which approximates with other study (13). Most patients $(83.3 \%)$ in current study were male. Large scale epidemiological studies and national cancer registries showed that male to female ratio varied depending of site of tumour from 2:1 to 15:1(14). $\mathrm{HNC}$ in women has been increased significantly in recent days, probably due to changes in 
environmental exposure (15). In the present study, the most common involved site was larynx (37.7\%) followed by oral cavity ca (27.6\%). This finding is in agreement with other reports (16) but it is not matched with studies where oral cavity is the most affected site (17).

In the current study, the median duration of symptoms in most cases were 5 months. Delay in diagnosis may be due to illiteracy or poor financial status, or tendency of most patients to resort to home remedies before seeking medical help. A comparable time span has been reported by Shenoi et al., in their study (18). In the present study, microscopically, Grade II (moderately differentiated) tumours were the most common (64.4\%) followed by Grade I (well differentiated) tumours (23.3\%).

In present study, ECOG PS ( $\mathrm{p}<0.001)$, smoking $(\mathrm{p}=$ $0.006)$, stage $(p=0.007)$, tumour grade $(p=0.041)$, NLR $(p<0.001)$ and PLR $(p=0.001)$ are the most important factor for the LRR and ECOG PS ( $\mathrm{p}<$ $0.001)$, smoking $(\mathrm{p}=0.020)$, stage $(\mathrm{p}=0.019)$, NLR $(p<0.001)$, and PLR ( $p<0.001)$ influencing the OS. This is supported by another study from India where 400 patients of oral cavity squamous cell carcinoma were analysed and showed advanced $\mathrm{T}$ stage $(P<$ $0.02)$, thicker tumours $(P<0.00)$ as important factor for LRR (19).

Normal NLR values are in the range of 0.78-3.53 in the general and NLR and PLR cut-off levels deployed in numerous studies varied in a wide range of PLR: 105.3-170 and NLR: 2.0 to 4.2 (20). In our study, the optimal threshold value, determined with the statistical method detailed above, was 3.9 for NLR and158.3 for PLR. The frequency of distribution of NLR risk group i.e., low risk NLR and high risk NLR were $52.9 \%$ and $47.1 \%$ respectively.

In the present study, we found NLR as one of the important prognostic factors for LRR and OS. Our findings matched with other studies $(19,20)$. Cho et al., analysed 621 patients and found the 5-year PFS and OS rates for all patients were $63.8 \%$ and $72.9 \%$, respectively. Both PFS and OS were significantly worse in high NLR patients receiving any type of treatment (PFS: $39.2 \%$ vs. $75.8 \%, p<0.001$; OS: $50.9 \%$ vs. $83.8 \%, p<0.001)$. In survival analyses, a high NLR remained significantly associated with a poor PFS and OS. The multivariate analysis revealed significant associations of a high NLR with poor PFS, an older age, and an advanced T classification. An elevated PLR was also found to be associated with a poor PFS (20). The patients with higher NLR showed more than 2.5 times higher risk of mortality compared to normal NLR (HR 2.62; 95\% CI 1.19$5.81 ; p=0.02)$. NLR $>5$ had a significant effect on survival as compared to those with NLR $<2.5$ ( $P<$ $0.016)$ for the group which received adjuvant chemoradiotherapy shown by Malik et al. (19). Bojaxhiu et al., analysed 186 patients treated from
2007 to 2010 with a result of higher NLR related with poor Overall Survival (21). In another study it has been shown that NLR values higher than the threshold i.e., 3.9 represented an enhanced risk regarding OS (22). Tazeen et al. showed a statistically significant association of PLR $(P=.001)$ and NLR $(P=.002)$ with shorter disease-free survival (DFS) (23). Sano et al. analysed 94 patients of locally advanced oral squamous cell carcinoma which demonstrated that high NLR and high PLR were associated with significantly worse OS and CSS (24). But our study result is not matched with their other results where they showed no association with high NLR with LRFS (HR, 1.49 (0.83$2.68), p=0.182), \quad$ DRFS $\quad(\mathrm{HR}, \quad 1.38 \quad(0.65-$ $3.22), p=0.4)$, or acute toxicity grade $\geq 2$ (24).

PLR also came out as important negative prognostic factor for survival in this study as also supported by other studies $(19,23)$. Malik et al. associated high PLR with higher frequency of various adverse clinicopathological factors such as higher pT stage $(P<0.00)$, nodal positivity $(P<0.036)$, poor grade of tumour $(P<0.00)$, thicker tumours $(P<0.00)$, presence of extra capsular extension (ECE) $(P<$ $0.04)$, perineural invasion (PNI) $(P<0.001)$, and positive margins ( $\mathrm{p}<0.001)$. Thus, higher PLR values were associated with increased frequency of these adverse clinicopathological factors. PLR was also found to have a significant negative impact on survival (19). Tazeen et al. showed in a multivariate analysis that a high PLR (HR, 2.998; 95\% CI, 1.128 to $7.968 ; P=0.028$ ) was independent factors for determining DFS and OS in oral cavity cancer (23). Ninety-two patients with locally advanced oropharyngeal cancer with a median follow-up was 39 months. Median NLR was 3 and median PLR was 153. The 3-year DFS was $82.9 \%$. Univariate analysis showed that PLR were statistically significant factors in determining 3-year DFS $(\mathrm{p}=0.01)$. High NLR and high PLR were correlated with lower DFS, patients with NLR > 4 had a 3 -years DFS of $50 \%$ versus $75 \%$ in the group with NLR $\leq 4(p=0.04)$ and patients with PLR > 150 had a 3 -years DFS of $65 \%$ versus $82 \%$ in the group with PLR $\leq 150(\mathrm{p}=0.04)(25)$.

In contrast to this, there were many studies which were not matched with our result where PLR was not associated with outcome with no significant correlation between baseline PLR and OS or the association between PLR and OS or DFS was not statistically significant (21).

Our results are therefore consistent with the concept that a high NLR contributes to poor disease control by suppressing the cytolytic activities of activated effector $\mathrm{T}$ cells and the peritumoral infiltration of immunosuppressive cells such as macrophages (26).

We further demonstrated that a higher PLR was associated with PFS in our multivariate analysis. Again, the significance of interactions between 
platelets and the tumour microenvironment remains somewhat unclear. The platelet count provides an additional index of systemic inflammation elicited by the tumour and degranulation. This inflammation with consequent release of platelet-derived proangiogenic mediators within the microvasculature of tumour serve as important factor for tumour growth (27).

\section{CONCLUSION}

Overall, the findings of the present study support that NLR and PLR are the potential biomarkers for host response to tumour aggressiveness, therapeutic response to chemo-radiotherapy and survival in HNC patients. NLR can be strongly used as biomarker for prognostication for outcome and survival of patients with head neck cancer. However, a well-designed, larger studies with longer follow-up is warranted.

\section{CONFLICT OF INTEREST}

Authors declare that there is no conflict of interest.

\section{REFERENCES}

1. Grégoire, V., Lefebvre, J. L., Licitra, L., Felip, E. EHNS-ESMO-ESTRO Guidelines Working Group. Squamous cell carcinoma of the head and neck: EHNSESMO-ESTRO Clinical practice guidelines for diagnosis, treatment and follow-up. Ann. Oncol. 2010; 21(5): 184-186.

2. GLOBOCAN 2012 (IARC) Section of Cancer Surveillance. [Last accessed on 2016 Jun 23]. http://globocan.iarc.fr/Pages/fact_sheets_population.as px

3. Hanahan, D., Weinberg, R. A. Hallmarks of cancer: the next generation. Cell. 2011; 144(5): 646-674.

4. Balkwill, F., Mantovani, A. Inflammation and cancer: back to Virchow? Lancet. 2001; 357: 539-545.

5. Atzpodien, J., Royston, P., Wandert, T., Reitz, M. Group D.G.C.R.C.C.-I.T. Metastatic renal carcinoma comprehensive prognostic system. Br. J. Cancer. 2003; 88: 348-353. doi: 10.1038/sj.bjc.6600768.

6. Rosculet, N., Zhou, X.C., Ha, P., Tang, M., Levine, M.A., Neuner, G., Califano J. Neutrophil-tolymphocyte ratio: Prognostic indicator for head and neck squamous cell carcinoma. Head Neck. 2017; 39: 662-667. doi: 10.1002/hed.24658.

7. Demers, M., Wagner, D. D. Neutrophil extracellular traps: a new link to cancer-associated thrombosis and potential implications for tumor progression. Oncoimmunology. 2013; 2(2): e2294614.

8. Buergy, D., Wenz, F., Groden, C., Brockmann, M. A. Tumor-Platelet interaction in solid tumors. Int. J. Cancer. 2012; 130: 2747-2760. doi: 10.1002/ijc.27441.

9. Ozawa, T., Ishihara, S., Nishikawa, T., Tanaka, T., Tanaka, J., Kiyomatsu, T.et al. The preoperative platelet to lymphocyte ratio is a prognostic marker in patients with stage II colorectal cancer. Int J Colorectal Dis 2015; 30: 1165-1171.

10. Edge, S. B., Compton, C. C., Fritz, A. G., Green, F. L. AJCC cancer staging manual. Springer, New York. 2012; 7.

11. Oken, M., Creech, R., Tormey, D., Horton, J., Davis, T., McFadden, E., et al. Toxicity and response criteria of the Eastern Cooperative Oncology Group; https://ecog-acrin.org/resources/ecog-performancestatus. (Accessed 3rd January 2015)

12. Coussens, L. M., Werb, Z. Inflammation and cancer. Nature. 2002; 420(6917): 860.
13. Bhattacharjee, A., Chakraborty, A., Purkaystha, P. Prevalence of head and neck cancers in the North Eastan institutional study. Indian Journal of Otolaryngology and Head and Neck Surgery. 2006; 58(1): 15-19.

14. Mehanna, H., Paleri, V., West, C., Nutting, C. Head and neck cancer-Part 1: Epidemiology, presentation, and prevention. BMJ. 2010; 341(7774): 663-666.

15. Alvarenga, L. D. M., Ruiz, M. T., Pavarino-Bertelli, É.C., Ruback M., Maniglia J., Goloni-Bertollo, M. Epidemiologic evaluation of head and neck patients in a university hospital of Northwestern São Paulo State. RevistaBrasileira de Otorrinolaringologia. 2008; 74(1): 68-73.

16. Andisheh-Tadbir, A., Ashrafi, M., Jafari-Ashkavandi, Z., Paknahad, M., Taheri, F. Head and neck squamous cell carcinoma in Southern Iran. Iran Red Crescent Med J. 2008; 10: 309-313.

17. Addala, L., Pentapati, C. K., Reddy, K., Thavanati, P. K., Anjaneyulu, V., Sadhnani, M. D. Risk factor profiles of head and neck cancer patients of Andhra Pradesh, India. Indian J Cancer 2012; 49: 215-219.

18. Shenoi, R., Devrukhkar, V., Chaudhuri, K., Sharma, B. K., Sapre, S. B., Chikhale, A., et al., Demographic and clinical profile of oral squamous cell carcinoma patients: A retrospective study. Indian J Cancer. 2012; 49: 21-26.

19. Malik, A., Mishra, A., Mair, M., Chakrabarti, S. Role of neutrophil-to-lymphocyte ratio and platelet-tolymphocyte ratio as prognostic markers in oral cavity cancers. Indian Journal of Medical and Pedriatic Oncology. 2019; 40(1): 94-100.

20. Cho, Y., Kim, J. W., Yoon, H. I. The prognostic significance of neutrophil-to-lymphocyte ratio in head and neck cancer patients treated with radiotherapy. J Clin Med.2018; 7(12): 512.

21. Bojaxhiu, B., Arnoud, J., Templeton, M., Elicin, O., Shelan, M. Relation of baseline neutrophil-tolymphocyte ratio to survival and toxicity in head and neck cancer patients treated with (chemo-) radiation. Radiation Oncology. 2018; 13: Article number: 216.

22. Szilasi, Z., Jósa, V., Zrubka, Z., Mezei, T., Vass, T., Merkel, K. et al. Neutrophil-to-lymphocyte and platelet-to-lymphocyte ratios as prognostic markers of survival in patients with head and neck tumoursresults of a retrospective multicentric study. Int J Enviorn Res Public Health. 2020; 17(5): 1742.

23. Tazeen, S., Prasad, K., Harrish, K., Sagar, P., Kapali, A. S., Chandramouli, S. Assessment of Pre-treatment Neutrophil/Lymphocyte Ratio and Platelet/Lymphocyte Ratio in Prognosis of Oral Squamous Cell Carcinom. Journal of Oral and Maxillofacial Surgery. 2020; 78(6): 949-60

24. Sano, Y., Kogashiwa, Y., Araki, R., Enok, Y., Ikeda, T., Yoda, T. et al. Correlation of inflammatory markers, survival, and COX2 expression in oral cancer and implications for prognosis. Otolaryngology- Head and Neck Surgery. 2018; 158(4): 667-676.

25. Espeli, V. G., Martucci, F., Richetti, A., Pastore, A. Prognostic neutrophil-to-lymphocyte and platelet-tolymphocyte ratio in locally advanced oropharyngeal cancer: a retrospective experience of two institutions. JCO. 2019; 37 (15) Suppl.

26. Mantovani, A., Schioppa, T., Porta, C., Allavena, P., Sica, A. Role of tumor-associated macrophages in tumor progression and invasion. Cancer Metastasis Rev. 2006; 25: 315-322. doi: 10.1007/s10555-0069001-7.

27. Sierko, E., Wojtukiewicz, M. Z. Inhibition of platelet function: Does it offer a chance of better cancer progression control? Semin. Thrombosis Hemost. 2007; 33: 712-721. doi: 10.1055/s-2007-991540. 